\title{
Bioinformatics Analysis Reveals CCR7 As A Potential Biomarker for Predicting CKD Progression
}

\author{
Junju Lai \\ Division of Nephrology, The First Affiliated Hospital of Guangzhou Medical University, Guangzhou \\ Huizhi Shan \\ Division of Nephrology, The First Affiliated Hospital of Guangzhou Medical University, Guangzhou \\ Sini Cui \\ Division of Nephrology, The First Affiliated Hospital of Guangzhou Medical University, Guangzhou \\ Lingfeng Xiao \\ Division of Nephrology, The First Affiliated Hospital of Guangzhou Medical University, Guangzhou \\ Xiaowen Huang \\ Division of Nephrology, The First Affiliated Hospital of Guangzhou Medical University, Guangzhou \\ Yun Xiao ( $\nabla$ msxiaoyun@sina.com ) \\ Division of Nephrology, The First Affiliated Hospital of Guangzhou Medical University, Guangzhou
}

\section{Research Article}

Keywords: Chronic kidney disease, Biomarker, CCR7

Posted Date: September 7th, 2021

DOI: https://doi.org/10.21203/rs.3.rs-730388/v1

License: (c) (1) This work is licensed under a Creative Commons Attribution 4.0 International License. Read Full License 


\section{Abstract \\ Background}

Chronic kidney disease (CKD) inevitably progresses to end-stage renal disease if intervention does not occur in time. However, there are limitations in predicting the progression of CKD by solely relying on changes in renal function. A biomarker with high sensitivity and specificity that can predict the progression of CKD early is required.

\section{Methods}

We used the online Gene Expression Omnibus (GEO) microarray dataset GSE45980 to identify differentially expressed genes (DEGs) in patients with progressive and stable CKD. We then performed functional enrichment and protein-protein interaction (PPI) network analysis on DEGs and identified key genes. Finally, the expression patterns of the key genes were verified using the GSE60860 data set, and the receiver operating characteristic curve analysis was performed to clarify their predictive ability of progressive CKD. Ultimately, we verified the expression profiles of these hub genes in an in vitro renal interstitial fibrosis model by RT-PCR and western blot analysis.

\section{Results}

Differential expression analysis identified 50 upregulated genes and 47 downregulated genes. The results of the functional enrichment analysis revealed that the upregulated DEGs were mainly enriched in immune response, inflammatory response, and NF-KB signaling pathways, whereas the downregulated DEGs were mainly related to angiogenesis and the extracellular environment. PPI network and key gene analysis identified CCR7 as the most important gene. CCR7 mainly plays a role in immune response, and its only receptors, CCL 19 and CCL21, have also been identified as DEGs. The ROC curve analysis of CCR7, CCL 19 and $C C L 21$ found that CCR7 and CCL 19 present good disease prediction ability.

\section{Conclusion}

CCR7 may be a stable biomarker for predicting the progression of CKD, and the CCR7-CCL 19/CCL21 axis may be a therapeutic target for end-stage renal disease. However, further experiments are needed to explore the relationship between these genes and CKD.

\section{Introduction}

Chronic kidney disease (CKD) affects $8-16 \%$ of the world's population [1]. Multiple regional epidemiological surveys indicate that the prevalence of CKD in China is around $10.8 \%$ [2]. Despite the high incidence of this disease, the general public does not fully understand the role the kidneys play in the human body and the consequences of kidney dysfunction. Recent statistical surveys indicate that less than $5 \%$ of the worldwide population know the function of the kidneys. In China, only $12.5 \%$ of the population is aware of the existence and implications of CKD [2,3]. The rate of CKD progression is highly variable between individuals. In some patients, the renal function stabilizes and does not further deteriorate during several years (phase termed "stable CKD"), and in some cases it even improves over time ("reverse CKD"). Nonetheless, the majority CKD patients experience a decline in renal function over time ("progressive CKD").

Although some controversy exists around the need for early screening of CKD, it is undeniable that early intervention in CKD patients results in improved quality of life and reduces the consumption of medical resources [4]. Early studies suggested that glomerular filtration rate (GFR) decline follows a linear model, and that it could be used to predict when patients might reach end-stage renal disease (ESRD) and need renal replacement therapy [5, 6]. However, recent studies suggest that the progression of CKD is not linear, hindering prognosis prediction [7].

At present, it is still very difficult to predict or identify patients who will suffer from progressive CKD. This is caused by the lack of consensus on the definition of CKD progression, and more importantly, the lack of sensitive and specific biomarkers for early prediction of CKD progression. The most commonly used surrogate markers for CKD progression are proteinuria, serum creatinine levels and GFR, but these markers must be cautiously used as they present some limitations [8, 9]. Although recently some biomarkers have been considered as intermediate endpoints, such as renal injury molecules (KIM-1) [10], neutrophil gelatinase-associated protein (NGAL) [11] and soluble urokinase receptor (suPAR) [12], evidence about the utility of these markers in clinical practice is still lacking.

In this study, we used statistical analysis tools and data mining techniques to reveal gene patterns responsible for the progress of CKD. We mined biomarkers that can predict the progress of CKD. Here, we used the kidney tissue microarray dataset GSE45980 created by Rudnicki et al. to perform genome-wide gene expression analysis to study differentially expressed genes (DEGs) in patients with progressive CKD and stable CKD. Our research will help understand the genetic pattern of CKD progression and provide new insights for the clinical diagnosis and treatment of progressive CKD.

\section{Materials And Methods}

\subsection{Microarray Data}

The dataset GSE45980 with microarray expression data generated by Rudnicki et al. was downloaded from the Gene Expression Omnibus (GEO, https://www.ncbi.nlm.nih.gov/geo/). The dataset is based on the GPL13497 Agilent-026652 Whole Human Genome Microarray 4x44K v2 (Probe Name 
version) array platform. The data set contained information about 43 kidney tissue samples from patients with different types of CKD. Twelve of these patients reached ESRD or doubled serum creatinine during follow-up were defined as progressive CKD patients, and the other 31 samples that did not meet the above criteria were defined as stable CKD patients. The annotation file for GPL13497 is also downloaded from GEO.

\subsection{Differential Expression Analysis}

DEG in progressive and stable CKD samples was analyzed by the limma package in Bioconductor [13]. The P-value of DEGs was calculated by using the unpaired Student's t-test using the limma package. We retained genes with the following criteria: (1) $\mid \log _{2}$ FoldChange| $>0.5$, and $(2)$ Adjusted P-values $<0.05$. The heatmap package was used to draw the heatmap of the DEGs in R.

\subsection{Functional Enrichment Analysis of DEGs}

We used the online tool David 6.8 (https://david.ncifcrf.gov/tools.jsp) to analyze the functional categories in which the DEGs were enriched [14]. The functional categories Gene Ontology (GO) terms and Kyoto Encyclopedia of Genes and Genomes (KEGG) pathways were used for the enrichment analyses. $\mathrm{GO}$ is an commonly used tool for gene annotation that classifies the genes using a defined, structured and controlled vocabulary in three distinct categories, namely molecular function (MF), biological process (BP) and cellular components (CC) [15]. The KEGG is a database for assigning DEG groups to specific pathways [16]. We selected only genes with $P$-value $<0.05$.

\subsection{Protein-Protein Interaction (PPI) Network Analysis}

PPI network analysis was performed using STRING (https://string-db.org/), an online database of known and predicted PPI [17]. We mapped DEGs to the PPI network using an interaction score $>0.4$ as the threshold. The software of Cytoscape v3.6.0 was used to visualize and construct the PPI network [18]. Then, using the plug-in cytohubba we selected the hub genes by calculating and analyzing the network structure and the weighted connection between nodes using 12 algorithms [19].

\subsection{Validation of the Hub Gene}

We used the dataset GSE60860 uploaded to GEO by Rudnicki et al., which contains renal tissue samples from 8 patients with progressive CKD and 21 patients with stable CKD. We annotated the samples using the array platform GPL13497 Agilent-026652 Whole Human Genome Microarray 4x44K v2 (Probe Name version). We then used the limma package to identify the genes differentially expressed. Statistical analyses were performed using SPSS v19.0 software (SPSS Inc., Chicago, IL, USA). Student's t-test was used for statistical comparison, and Pearson correlation analysis was used for correlation analysis using age and gender as covariables. The receiver operating characteristic (ROC) curve was used to reflect the area under the curve (AUC) of hub genes to screen markers and evaluate their critical values. The significance levels were set at $\mathrm{P}<0.05$.

\subsection{Cell culture}

NRK-52E cells were obtained from Jinan University (Guangdong, China) and were cultured in Dulbecco's Modified Eagle Medium (DMEM. GIBCO BRL, Life Technologies Inc., Gaithersburg, MD, USA) with $10 \%$ Fetal Bovine Serum (FBS, GIBCO BRL) at $37^{\circ} \mathrm{C}$ and $5 \% \mathrm{CO}$. NRK-52E cells were seeded in 6-well plates. When the cells confluence reached $60 \%-70 \%$, they were transferred to serum-free medium and starved for $24 \mathrm{~h}$. Recombinant human TGF- $\beta 1$ at a final concentration of $10 \mathrm{ng} / \mathrm{ml}$ was subsequently added to the culture medium, and cultured for $0,6,12$, and $24 \mathrm{~h}$.

\subsection{Quantitative real-time PCR}

Total RNA was extracted from NRK-52E cells using TRIzol reagent (Invitrogen) according to the manufacturer's instructions. cDNA was synthesized from equal amounts of RNA using reverse transcriptase purchased from Takara Bio Inc. (Shiga, Japan). The specific primers for the target genes were designed by GENEray (Shanghai, China) and synthesized according to the GeneBank sequences cDNA was amplified by PCR using specific pairs of primers. The reaction system was formulated in compliance with the specifications of SYBR Premix EX Taqkits (Takara bio Inc.). The reaction conditions were: pre denaturation at $95^{\circ} \mathrm{C}$ for $30 \mathrm{~s}$, denaturation at $95^{\circ} \mathrm{C}$ for $5 \mathrm{~s}$, annealing at $58^{\circ} \mathrm{C}$ to $60^{\circ} \mathrm{C}$ (depending on the specific pair of primers used), extension for $10 \mathrm{~s}$ for $40 \mathrm{cycles}$, and then annealing at $95^{\circ} \mathrm{C}$ for $15 \mathrm{~s}$. mRNA expression was calculated according to the standard curve, using expression levels of $G A P D H$ as the internal control. Primer sequences were as follows: CCR7 (forward: 5'-TGTACGAGTCGGTGTGCTTC-3'; reverse: 5'- GGTAGGTATCCGTCATGGTCTTG-3'), FN (forward: 5'GTGTCCTCCTTCCATCTTC-3'; reverse: 5'-CAGACTGTCGGTACTCACG-3'), GAPDH (forward: 5'- AGGTCGGTGTGAACGGATTTG-3'; reverse: 5'TGTAGACCATGTAGTTGAGGTCA-3').

\subsection{Protein extraction and western blot analysis}

NRK-52E cells were lysed with RIPA lysis buffer on ice and centrifuged. Protein concentrations were determined by enzyme-linked immunoassay (Bio-Rad) and equilibrated with RIPA buffer. Proteins were separated on 10\% SDS-PAGE gels and transferred to nitrocellulose membranes (Millipore, Billerica, MA, USA). Membranes were sealed in 5\% non-fat milk for $2 \mathrm{~h}$ at room temperature. After three times TBS-T washes, membranes were incubated with the corresponding primary antibodies (rabbit polyclonal anti-CCR7 antibodies (Proteintech, 1:1000), rabbit polyclonal anti-GAPDH, FN antibodies (Cell Signaling Technologies,

1:1000)) overnight at $4^{\circ} \mathrm{C}$. Then, membranes were rinsed three times with TBS-T, and incubated with horseradish peroxide intensified secondary antibodies for 2 h. Protein expression levels were detected and imaged generated using the Odyssey CLX Imaging System (LI-COR, Lincoln, NE, USA).

\subsection{Statistical analysis}

Data are presented as mean \pm standard error of mean (SEM) indicates. Statistical tests were performed using SPSS 16.0 statistical software (SPSS Inc., Chicago, IL, USA). Unpaired Student's t-test was used to compare means between two groups. One-way ANOVA and LSD test were used for comparison of multiple groups. P-values less than 0.05 were considered statistically significant. 


\section{Results}

\subsection{Genes differentially expressed between progressive and stable CKD patient samples.}

We downloaded the microarray expression dataset GSE45980 from the GEO database and analyzed the DEGs in progressive and stable CKD samples. A total of 50 upregulated genes and 47 downregulated genes were identified between patients with progressive and stable CKD (Fig. 1). The top ten significantly upregulated and downregulated genes are listed in Table 1 ranked by $\log _{2}$ FoldChange value.

\subsection{Functional and Pathway Enrichment of DEGs}

We then analyzed the pathway and functions in which the identified DEGs were enriched. A total of 22 significantly enriched GO terms and 4 KEGG pathways were identified. The upregulated DEGs were mainly enriched in the $\mathrm{GO}$ terms related to the immune response, inflammatory response, and NF-KB signaling pathway, whereas the downregulated DEGs were mainly related to angiogenesis and the extracellular environment (Table 2). In addition, the upregulated DEGs were in the KEGG pathways chemokine signaling pathway, cytokine-cytokine receptor interaction, and NF-KB signaling pathway. Down-regulated DEGs were significantly enriched in vascular smooth muscle contraction (Table 3).

\subsection{PPI Network Analysis of DEGs}

A PPI network with 55 nodes and 116 edges, and interaction score of the network $>0.4$ was generated using the tools STRING and Cytoscape (Fig. $2 A$ ). In the generated network, each node represents one gene, and edges represent links between genes. Up-regulated genes are depicted in red, whereas downregulated genes are depicted in green. Analysis of the key modules of the network revealed that there were two main modules, which included five upregulated genes

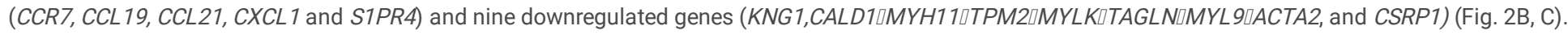
The hub genes were then determined using 12 distinct calculation methods (Table 4). The gene with the highest repetition rate was $C C R 7$ (in the 12 calculation methods, 10 times appeared in the top 10 genes).

\subsection{Identification of Genes of Interest and Validation of the several DEGs using other datasets}

The only ligands of the protein encoded by CCR7, which was identified as the most important gene, are CCL19 and CCL21 [20]. Both CCL 19 and CCL21 were identified as upregulated DEGs. Enrichment analysis showed that these three genes were enriched in immune response, inflammatory response, and NF-KB signaling pathway, all of which are important pathways for the progression of CKD to end-stage renal disease.

To verify the expression levels of the three genes identified as potentially important DEGs in the progression to end-stage renal disease, we analyzed their expression levels in the dataset GSE60860. In this dataset, a total of 154 DEGs were identified. We closely monitored the expression levels of $C C R 7(\mathrm{P}<0.05)$, CCL21 $(\mathrm{P}<0.05)$, and $C C L 19(\mathrm{P}<0.05)$, and compared their expression in the dataset (Fig. 3A). Spearman correlation analysis showed that CCR7, CCL21 and CCL 19 expression levels were not significantly correlated with age and gender (Table 5).

In addition, we evaluated the potential utility of using CCR7, CCL21, and CCL 19 expression levels as biomarkers using ROC curves generated with the two datasets (Fig. 3B). The AUC of CCR7 was $0.767(\mathrm{P}<0.01,95 \% \mathrm{Cl}=0.645-0.889)$, and the sensitivity and specificity of predicting $C K D$ progression were 0.65 and 0.788 , respectively. The AUC of $C C L 19$ was $0.755(P<0.001,95 \% \mathrm{Cl}=0.635-0.874)$, and the sensitivity and specificity were 0.900 and 0.635 . Finally, the AUC of CCL21 was $0.629(P>0.05)$. The results showed that both CCR7 and CCL 19 had a good predictive ability. The lower AUC of CCL21 might be caused by the number of samples.

\subsection{Validation of CCR7 expression in an in vitro renal interstitial fibrosis model.}

To further validate the relationship between $C C R 7$ expression levels and CKD severity, we examined the expression of $C C R 7$ using an in vitro model of renal interstitial fibrosis. We used TGF- $\beta 1$-stimulated NRK-52E cells to model renal interstitial fibrosis. To model the progression of CKD to end-stage renal disease, we used longer TGF- $\beta 1$ stimulation times. Analysis of the mRNA and protein expression levels using RT-qPCR and Western Blot revealed that the protein and mRNA expression levels of FN and CCR7 gradually increased with the duration of TGF- $\beta 1$ stimulation. The observed increased was especially notable at $24 \mathrm{~h}$ and $48 \mathrm{~h}$ (Fig. 4). The expression levels of FN and CCR7 were positively correlated.

\section{Discussion}

In this study, 97 DEGs, of which 50 were upregulated and 47 were downregulated, were identified in progressive CKD samples compared with stable CKD samples in the array dataset GSE45980. Enrichment analysis showed that upregulated DEGs were significantly enriched in pathways related to the immune response, inflammatory response, and NF-KB signaling pathway, which was also recognized as the key process for the progression of CKD to end-stage renal disease. The gene CCR7, which was upregulated in progressive CKD samples, was enriched in the above GO-terms and KEGG pathways, and presented the highest interaction degree in the PPI network. Using an in vitro model of renal interstitial fibrosis model, we identified that the expression level of CCR7 was concomitantly increased with the aggravation of oxidative stress.

Chemokines play a key role in numerous immune processes, including immune cell development, immune response initiation, and the pathophysiological recruitment of immune cells in infections and diseases. Functionally, chemokines can be divided into two categories: inflammatory chemokines, which are mainly expressed during activation of the immune response, and steady-state chemokines, which are constitutively expressed in discrete locations without obvious activation stimuli [21]. Nonetheless, most chemokines have both functions. CCR7 is an essential chemokine necessary to maintain homeostasis. Under homeostatic conditions, low levels of CCR7 in the lymph nodes contributes to peripheral tolerance. However, when encountering inflammatory mediators, pathogens or tissue damage, dendritic cells (DCs) express high amounts of CCR7, which in turn induces an immune response [20, 22].

Page $4 / 12$ 
CCR7 has only two constitutive ligands, CCL19 (also known as Eb11 ligand chemokine or ELC) and CCL21 (also known as secondary lymphochemokine or SLC). CCL21 has a distinctive 32 amino acids-long C-terminal tail. Twelve of the C-terminal amino acids are basic amino acid residues that can bind to glycosaminoglycans and other molecules [23]. Whereas CCL21 mediates the migration effects in CCR7, CCL19 plays a complementary role and mediates nonmigration signals, such as promoting cell survival [24]. Both these ligands, CCL21 and CCL19, were also identified as upregulated DEGs.

The CCR7-CCL19/CCL21 axis can promote the retention of CD4 ${ }^{+}$T lymphocytes at the reconstruction site of collateral arteries, thereby promoting arteriogenesis [25]. Liu et al. found that Baicalin inhibited the levels of CCR7 and NF-KB simultaneously when studying the anti-inflammatory mechanism of Baicalin in asthma [26]. Qian et al. found that Newcastle disease virus-like particles (NDV VLPS) activate DCs through TLR4/NF-KB pathway, and promote DC migration through the CCR7-CCL19/CCL21 axis [27]. These results show that CCR7-CCL19/CCL21 axis may be related to the NF-KB pathway, which plays an important role in the cellular inflammatory response and immune response [28]. In addition, McNamee et al. found that CCL19, CCL21 and CCR7 were involved in the induction and maintenance of chronic inflammation in a Crohn's-like ileitis mice model [29]. These results are consistent with the results of the GO term and KEGG pathways enrichment analysis.

Several studies have found that CCL21 and CCR7 are expressed in interstitial fibroblasts and tubular epithelial cells in transplanted kidneys [30], and the CCL21-CCR7 axis can promote renal fibrosis [31]. Early studies have shown that lymph angiogenesis is closely related to the progression of tubulointerstitial fibrosis [32]. Pei et al. identified that that lymphatic vessels were significantly increased in CKD biopsy samples relative to normal samples [33]. In addition, CCL21 was highly expressed in lymphatic vessels, with CCR7 ${ }^{+}$lymphocytes and DCs clustered near lymphatic vessels. The same results were observed in unilateral ureteral obstruction animal models, in which blocking CCR7 inhibits lymph angiogenesis and reduces injury-induced inflammation and fibrosis. These results suggest that the recruitment of lymphocytes and DC in the kidney depends on the CCL21-CCR7 axis. Altogether, these results indicate that continuous damage to the kidney leads to lymphatic vessels angiogenesis and the recruitment CCR7 ${ }^{+}$cells into the kidney, which in turn and accelerates intrarenal inflammation. This suggests that there is a relationship between the expression level of CCR7 and the severity of CKD. Studies have found that CCL19 can inhibit cell viability and promote cellular inflammation and fibrosis in DN cells [34]. Mesenchymal stem cells can also inhibit renal inflammation by reducing the expression levels of CCL19 [35]. At present, there are few reports about CCL19, since the CCL19-CCR7 axis is commonly overlooked in comparison with the CCL21-CCR7 axis. However, recent reports show that the CCL19-CCR7 axis plays an important role in renal inflammation. Limited sample data and related experimental results show that, compared with CCR7, CCL19 and CCL21 are still unstable markers for predicting the progress of CKD.

In conclusion, CCR7 may play an important role in the progression of CKD through the immune system. The expression level of CCR7 may be related to the severity of CKD. We found that the expression level of CCR7 was independent of gender and age, indicating that it may be a prognostic marker for CKD progression in individuals of different age and gender. However, more experiments and larger multicenter prospective studies are required to validate the utility of this marker, as well as the threshold for predicting CKD progression and adverse events. As the only ligands of CCR7, the roles of CCL19 and CCL21 in the progression of CKD still need further study. Recent results indicate that the CCR7-CCL19/CCL21 axis may be a therapeutic target for chronic kidney disease and end-stage renal disease.

\section{Declarations}

\section{Ethics approval and consent to participate}

The datasets from the GEO database has been approved by the Institutional Review Board of the Medical University of Innsbruck.

\section{Consent for publication}

Not applicable.

\section{Availability of data and materials}

The datasets in the current study come from CEO database: GSE45980 and GSE60860.

\section{Competing interests}

The authors declare that they have no competing interests.

\section{Funding}

National Natural Science Foundation of China. (Grant No. 81600538)

\section{Authors' contributions}

J.L. designed the study, Y.X. supervised the study, J.L. and H.S. drafted the manuscript, S.C., L.X. and X.H. collected data. The author(s) read and approved the final manuscript.

\section{Acknowledgements}

We would like to thank Editage (www.editage.cn) for English language editing.

\section{References}


1. Chen, T.K., D.H. Knicely and M.E. Grams, Chronic Kidney Disease Diagnosis and Management: A Review. JAMA, 2019. 322(13): p. 1294-1304.

2. Zhang, L., et al., Prevalence of chronic kidney disease in China: a cross-sectional survey. Lancet, 2012. 379(9818): p. 815 - 22.

3. Tuot, D.S., et al., Chronic Kidney Disease Awareness Among Individuals with Clinical Markers of Kidney Dysfunction. Clinical Journal of the American Society of Nephrology, 2011. 6(8): p. 1838-1844.

4. Levin, A. and P.E. Stevens, Early detection of CKD: the benefits, limitations and effects on prognosis. Nat Rev Nephrol, $2011.7(8)$ : p. 446 - 57.

5. Mitch, W.E., et al., A simple method of estimating progression of chronic renal failure. Lancet, 1976. 2(7999): p. $1326-8$.

6. Modification Of Diet In Renal Disease Study Group, et al., Predictors of the progression of renal disease in the Modification of Diet in Renal Disease Study. Kidney International, 1997. 51(6): p. 1908-1919.

7. Li, L., et al., Longitudinal Progression Trajectory of GFR Among Patients With CKD. American Journal of Kidney Diseases, $2012.59(4)$ : p. 504-512.

8. Glassock, R.J., Debate: CON Position. Should Microalbuminuria Ever Be Considered as a Renal Endpoint in Any Clinical Trial. American Journal of Nephrology, 2010. 31(5): p. 462-465.

9. Coresh, J., et al., Decline in Estimated Glomerular Filtration Rate and Subsequent Risk of End-Stage Renal Disease and Mortality. JAMA, 2014. 311(24): p. 2518.

10. Sabbisetti, V.S., et al., Blood kidney injury molecule-1 is a biomarker of acute and chronic kidney injury and predicts progression to ESRD in type I diabetes. Journal of the American Society of Nephrology, 2014. 25(10): p. 2177-2186.

11. Bolignano, D., et al., Neutrophil Gelatinase-Associated Lipocalin (NGAL) and Progression of Chronic Kidney Disease. Clinical Journal of the American Society of Nephrology, 2009. 4(2): p. 337-344.

12. Hayek, S.S., et al., Soluble Urokinase Receptor and Chronic Kidney Disease. N Engl J Med, 2015. 373(20): p. $1916-25$.

13. Ritchie, M.E., et al., limma powers differential expression analyses for RNA-sequencing and microarray studies. Nucleic Acids Research, 2015. 43(7): p. e47-e47.

14. Huang, D.W., B.T. Sherman and R.A. Lempicki, Systematic and integrative analysis of large gene lists using DAVID bioinformatics resources. Nat Protoc, 2009. 4(1): p. 44-57.

15. Ashburner, M., et al., Gene Ontology: tool for the unification of biology. Nature Genetics, 2000. 25(1): p. 25-29.

16. Kanehisa, M. and S. Goto, KEGG: kyoto encyclopedia of genes and genomes. Nucleic Acids Res, 2000. 28(1): p. 27-30.

17. Szklarczyk, D., et al., The STRING database in 2017: quality-controlled protein-protein association networks, made broadly accessible. Nucleic Acids Research, 2017. 45(D1): p. D362-D368.

18. Shannon, P., et al., Cytoscape: a software environment for integrated models of biomolecular interaction networks. Genome Res, 2003. 13(11): p. 2498 504.

19. Chin, C., et al., cytoHubba: identifying hub objects and sub-networks from complex interactome. BMC systems biology, 2014.8 Suppl 4(Suppl 4): p. S11.

20. Förster, R., A.C. Davalos-Misslitz and A. Rot, CCR7 and its ligands: balancing immunity and tolerance. Nature Reviews Immunology, 2008. 8(5): p. 362371.

21. Zlotnik, A. and O. Yoshie, The chemokine superfamily revisited. Immunity, 2012. 36(5): p. 705 - 16.

22. Ohl, L., et al., CCR7 governs skin dendritic cell migration under inflammatory and steady-state conditions. Immunity, 2004. 21 (2): p. 279 - 88.

23. Yoshida, R., et al., Secondary lymphoid-tissue chemokine is a functional ligand for the CC chemokine receptor CCR7. J Biol Chem, 1998. 273(12): p. 711822.

24. Comerford, I., et al., A myriad of functions and complex regulation of the CCR7/CCL19/CCL21 chemokine axis in the adaptive immune system. Cytokine Growth Factor Rev, 2013. 24(3): p. $269-83$.

25. Nossent, A.Y., et al., CCR7-CCL19/CCL21 Axis is Essential for Effective Arteriogenesis in a Murine Model of Hindlimb Ischemia. J Am Heart Assoc, 2017. $6(3)$.

26. Liu, J., et al., Baicalin attenuates inflammation in mice with OVA-induced asthma by inhibiting NF-kappaB and suppressing CCR7/CCL19/CCL21. Int J Mol Med, 2016. 38(5): p. 1541-1548.

27. Qian, J., et al., Newcastle disease virus-like particles induce DC maturation through TLR4/NF-kappaB pathway and facilitate DC migration by CCR7CCL19/CCL21 axis. Vet Microbiol, 2017. 203: p. 158-166.

28. Sun, S.C., The non-canonical NF-kappaB pathway in immunity and inflammation. Nat Rev Immunol, 2017. 17(9): p. 545-558.

29. McNamee, E.N., et al., Ectopic lymphoid tissue alters the chemokine gradient, increases lymphocyte retention and exacerbates murine ileitis. Gut, 2013. 62(1): p. 53-62.

30. Zhou, H.L., et al., Distribution and expression of fibroblast-specific protein chemokine CCL21 and chemokine receptor CCR7 in renal allografts. Transplant Proc, 2013. 45(2): p. $538-45$.

31. Sakai, N., et al., Secondary lymphoid tissue chemokine (SLC/CCL21)/CCR7 signaling regulates fibrocytes in renal fibrosis. Proc Natl Acad Sci U S A, 2006. 103(38): p. 14098-103.

32. Sakamoto, I., et al., Lymphatic vessels develop during tubulointerstitial fibrosis. Kidney Int, 2009. 75(8): p. 828 - 38.

33. Pei, G., et al., Lymphangiogenesis in kidney and lymph node mediates renal inflammation and fibrosis. Science advances, 2019. 5(6): p. eaaw5075.

34. Sun, J., et al., MiR-325-3p inhibits renal inflammation and fibrosis by targeting CCL19 in diabetic nephropathy. Clin Exp Pharmacol Physiol. 2020;47(11):1850-1860. 


\section{Tables}

Table 1

Top ten up- and downregulated gene

\begin{tabular}{|llllll|}
\hline \multicolumn{5}{|l}{ Upregulated genes } & \multicolumn{5}{l|}{ Downregulated genes } \\
\hline Gene symbol & logFC & adj.P.Val & Gene symbol & logFC & adj.P.Val \\
\hline TBC1D10C & 1.270903 & 0.012108 & ACTA2 & -1.283903 & 0.019401 \\
\hline REG1A & 1.266544 & 0.021584 & MYH11 & -1.198393 & 0.047796 \\
\hline CCL19 & 1.260658 & 0.047002 & KNG1 & -1.004037 & 0.033276 \\
\hline IL7R & 1.199639 & 0.029889 & DCN & -0.874742 & 0.043624 \\
\hline LTB & 1.164515 & 0.021584 & MGP & -0.872574 & 0.021584 \\
\hline JAK3 & 1.082178 & 0.012437 & LPL & -0.8698 & 0.04524 \\
\hline CCL21 & 1.07003 & 0.04524 & EMCN & -0.852292 & 0.04524 \\
\hline PIGR & 1.034732 & 0.041715 & TIMP3 & -0.847645 & 0.013315 \\
\hline CCR7 & 1.034619 & 0.021584 & TAGLN & -0.831096 & 0.021584 \\
\hline RASAL3 & 0.999338 & 0.010765 & TMEM204 & -0.830443 & 0.013315 \\
\hline
\end{tabular}


Table 2

GO analysis of differentially expressed genes $(P<0.05$, count $\geq 4)$.

\begin{tabular}{|c|c|c|c|c|}
\hline GO-ID & Term & Count & PValue & Genes \\
\hline \multicolumn{5}{|c|}{ Upregulated genes } \\
\hline \multicolumn{5}{|l|}{$\begin{array}{l}\text { GO-BP } \\
\text { terms }\end{array}$} \\
\hline GO:0006955 & immune response & 9 & 1.17E-05 & CXCL1, CCR7, TNFRSF25, CCL21, S1PR4, IRF8, CCL19, IL7R, LTB \\
\hline GO:0090023 & $\begin{array}{l}\text { positive regulation of neutrophil } \\
\text { chemotaxis }\end{array}$ & 4 & $2.50 \mathrm{E}-05$ & CXCL1, CCR7, CCL21, CCL19 \\
\hline G0:0006954 & inflammatory response & 7 & 4.34E-04 & CXCL1, CCR7, TNFRSF25, CCL21, RELB, CCL19, ADAM8 \\
\hline GO:0051897 & $\begin{array}{l}\text { positive regulation of protein } \\
\text { kinase B signaling }\end{array}$ & 4 & 0.001379 & CCR7, CCL21, CCL19, ADAM8 \\
\hline GO:0043123 & $\begin{array}{l}\text { positive regulation of I-kappaB } \\
\text { kinase/NF-kappaB signaling }\end{array}$ & 4 & 0.008592 & CCR7, CCL21, CCL19, BIRC3 \\
\hline GO:0007165 & signal transduction & 8 & 0.030044 & CXCL1, CLDN3, TNFRSF25, RASGRP2, RASAL3, IL7R, LTB, ARHGAP9 \\
\hline GO:0007166 & $\begin{array}{l}\text { cell surface receptor signaling } \\
\text { pathway }\end{array}$ & 4 & 0.034825 & TNFRSF25, CD2, BIRC3, IL7R \\
\hline \multicolumn{5}{|l|}{$\begin{array}{l}\text { GO-CC } \\
\text { terms }\end{array}$} \\
\hline GO:0005578 & proteinaceous extracellular matrix & 4 & 0.033472 & HAPLN3, CCL21, ADAMTS10, PAPLN \\
\hline \multicolumn{5}{|l|}{$\begin{array}{l}\text { GO-MF } \\
\text { terms }\end{array}$} \\
\hline GO:0005102 & receptor binding & 5 & 0.010352 & CXCL1, TMC8, CD2, JAK3, LTB \\
\hline G0:0008270 & zinc ion binding & 8 & 0.02123 & MICALL2, ZMIZ2, ZSWIM7, ADAMTS10, PAPLN, BIRC3, ADAM8, SP140 \\
\hline \multicolumn{5}{|c|}{ Downregulated genes } \\
\hline \multicolumn{5}{|l|}{$\begin{array}{l}\text { GO-BP } \\
\text { terms }\end{array}$} \\
\hline GO:0006936 & muscle contraction & 6 & $9.54 \mathrm{E}-06$ & ACTA2, CALD1, MYH11, TPM2, MYLK, MYL9 \\
\hline G0:0001525 & angiogenesis & 5 & 0.002945 & EMCN, EPAS1, TEK, MCAM, RAMP1 \\
\hline \multicolumn{5}{|l|}{$\begin{array}{l}\text { GO-CC } \\
\text { terms }\end{array}$} \\
\hline G0:0031012 & extracellular matrix & 8 & 7.43E-06 & LPL, NES, RARRES2, HTRA1, LGALS1, MGP, DCN, TIMP3 \\
\hline G0:0070062 & extracellular exosome & 18 & 2.17E-04 & $\begin{array}{l}\text { KNG1, TPPP3, LPL, RARRES2, ACTA2, LGALS1, C160RF89, MGP, CSRP1, } \\
\text { TIMP3, DUSP26, HTRA1, CLIC5, MYH11, VAMP5, PRSS23, MGAT5, MYLK }\end{array}$ \\
\hline GO:0005615 & extracellular space & 10 & 0.005019 & KNG1, DKK3, LPL, HTRA1, ACTA2, LGALS1, DCN, MCAM, TIMP3, RAMP1 \\
\hline GO:0005576 & extracellular region & 11 & 0.005093 & $\begin{array}{l}\text { KNG1, DKK3, LPL, EMCN, RARRES2, HTRA1, NELL1, TEK, ANTXR2, DCN, } \\
\text { TIMP3 }\end{array}$ \\
\hline GO:0009986 & cell surface & 6 & 0.010474 & LPL, LGALS1, TEK, VAMP5, RAMP1, AOC3 \\
\hline \multicolumn{5}{|l|}{$\begin{array}{l}\text { GO-MF } \\
\text { terms }\end{array}$} \\
\hline GO:0003779 & actin binding & 4 & 0.031639 & TAGLN, CALD1, TPM2, MYLK \\
\hline
\end{tabular}

Table 3

KEGG pathway enrichment analyses for differentially expressed genes $(P<0.05$, Count $\geq 4)$.

\begin{tabular}{|c|c|c|c|c|}
\hline ID & Description & Count & PValue & Genes \\
\hline \multicolumn{5}{|c|}{ Upregulated genes } \\
\hline hsa04062 & Chemokine signaling pathway & 7 & 1.39E-05 & CXCL1, CCR7, CCL21, RASGRP2, CCL19, JAK3, PLCB2 \\
\hline hsa04060 & Cytokine-cytokine receptor interaction & 7 & 6.34E-05 & CXCL1, CCR7, TNFRSF25, CCL21, CCL19, IL7R, LTB \\
\hline hsa04064 & NF-kappa B signaling pathway & 5 & $1.21 \mathrm{E}-04$ & CCL21, RELB, CCL19, BIRC3, LTB \\
\hline \multicolumn{5}{|c|}{ Downregulated genes } \\
\hline hsa04270 & Vascular smooth muscle contraction & 5 & 3.80E-04 & ACTA2, CALD1, RAMP1, MYLK, MYL9 \\
\hline
\end{tabular}




\begin{tabular}{|c|c|c|c|c|c|c|c|c|c|c|c|}
\hline \multicolumn{12}{|c|}{ Rank methods in cytoHubba } \\
\hline MCC & DMNC & MNC & Degree & EPC & BottleNeck & EcCentricity & Closeness & Radiality & Betweenness & Stress & ClusteringCoefficien \\
\hline TAGLN & ACTA2 & CCR7 & CCR7 & CCR7 & PLCB2 & PLCB2 & CCR7 & IKZF1 & TEK & KNG1 & JAK3 \\
\hline MYH11 & MYLK & CCL19 & S1PR4 & CCL19 & TEK & KNG1 & IKZF1 & KNG1 & KNG1 & TEK & LGALS1 \\
\hline ACTA2 & TPM2 & S1PR4 & IKZF1 & S1PR4 & KNG1 & RASGRP2 & S1PR4 & CCR7 & IKZF1 & IKZF1 & ARHGAP9 \\
\hline MYLK & MYL9 & IKZF1 & CCL19 & IKZF1 & IKZF1 & $\mathrm{DCN}$ & KNG1 & S1PR4 & PLCB2 & PLCB2 & CALD1 \\
\hline TPM2 & CSRP1 & TAGLN & TAGLN & KNG1 & TAGLN & PRSS23 & CCL19 & CCL19 & ACTA2 & S1PR4 & CSRP1 \\
\hline MYL9 & CALD1 & MYH11 & ACTA2 & CXCL1 & CCR7 & COX7A1 & IRF8 & PLCB2 & S1PR4 & ACTA2 & NES \\
\hline CSRP1 & KNG1 & ACTA2 & KNG1 & CCL21 & RUNX3 & TEK & CXCL1 & IRF8 & TAGLN & TIMP3 & MYL9 \\
\hline CALD1 & TAGLN & MYLK & MYH11 & RELB & TIMP3 & IKZF1 & CCL21 & CXCL1 & TIMP3 & CCR7 & TPM2 \\
\hline CCR7 & MYH11 & TPM2 & MYLK & $\mathrm{CD} 2$ & ACTA2 & CCR7 & WDFY4 & CCL21 & DCN & TAGLN & CCL21 \\
\hline CCL19 & CXCL1 & MYL9 & TPM2 & IRF8 & S1PR4 & TIMP3 & ACTA2 & TIMP3 & CCR7 & DCN & CXCL1 \\
\hline
\end{tabular}

MCC: Maximal cilque centrality; MNC:Maximum neighborhood component; Degree: Node connect degree; EPC: Edge percolated component.

Table 5

Correlation analysis of CCR7, CCL21, CCL19 with gender and age

\begin{tabular}{|c|c|c|c|c|c|}
\hline & & \multicolumn{2}{|l|}{ Age } & \multicolumn{2}{|l|}{ Gender } \\
\hline & & $\mathbf{r}_{\mathrm{s}}$ & p & $r_{s}$ & $p$ \\
\hline \multirow[t]{3}{*}{ Spearman's tho } & CCR7 & 0.109 & 0.361 & -0.155 & 0.195 \\
\hline & CCL21 & -0.172 & 0.148 & 0.1 & 0.403 \\
\hline & CCL19 & 0.143 & 0.23 & -0.003 & 0.977 \\
\hline$r_{s}$, Spearman's $r^{2}$ & correl & coef & & & \\
\hline
\end{tabular}

\section{Figures}




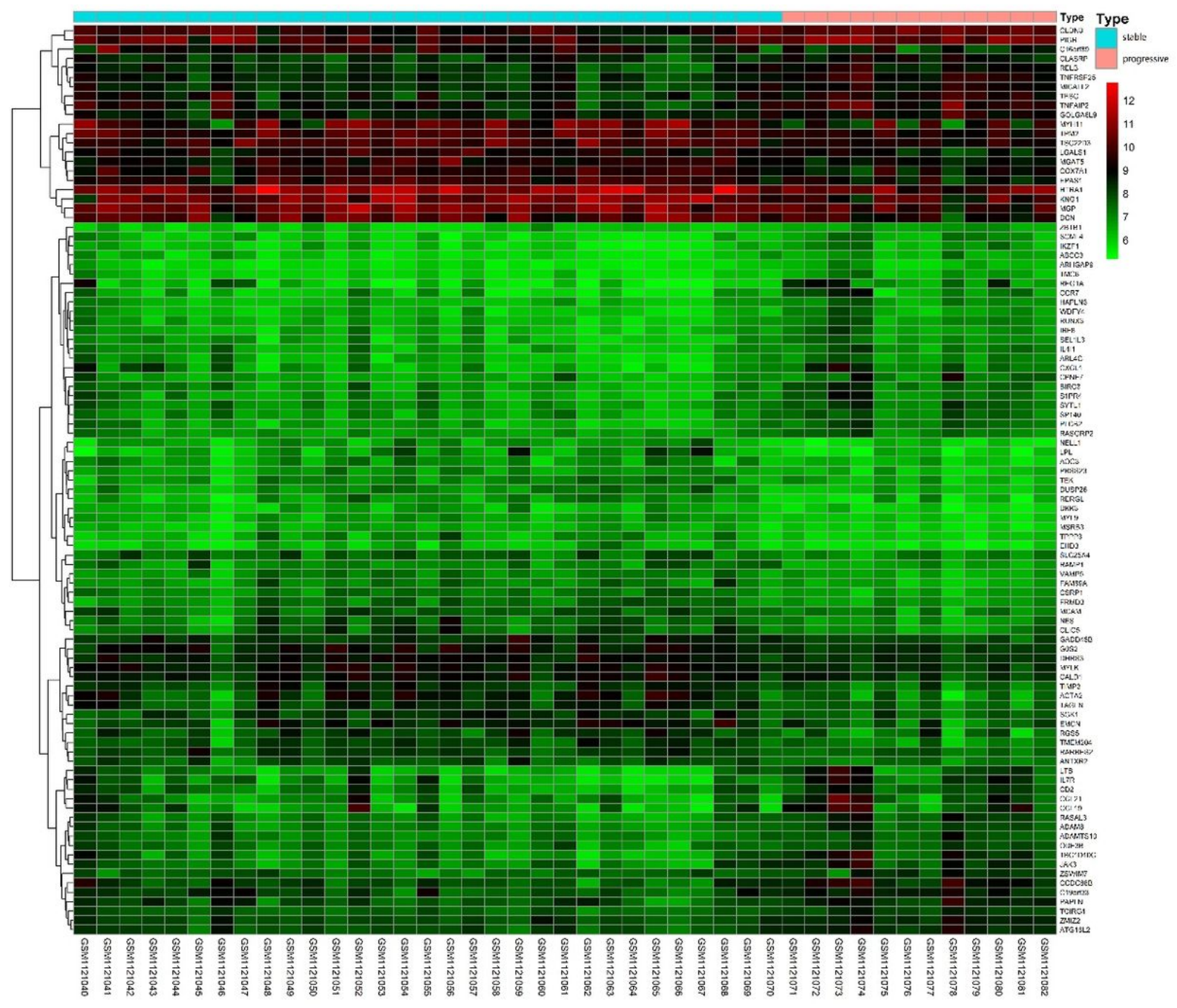

\section{Figure 1}

Heatmap of 97 differentially expressed genes were detected in 31 stable CKD patients and 12 progressive CKD patients. Red represents upregulated genes and the green represents downregulated genes. 


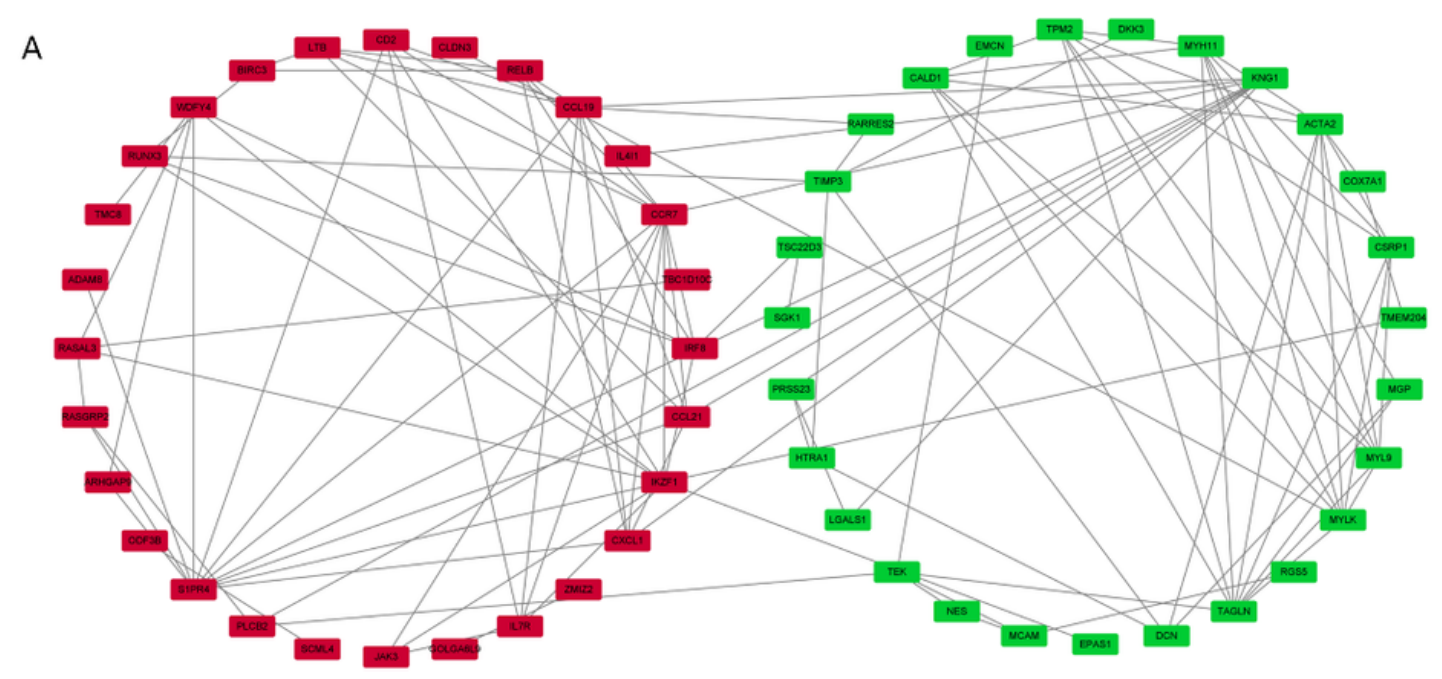

B

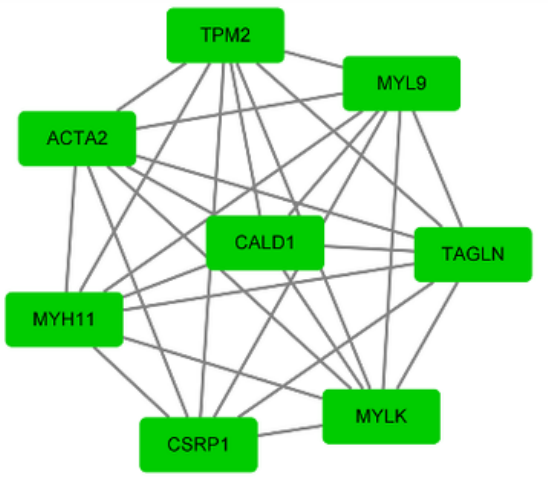

C

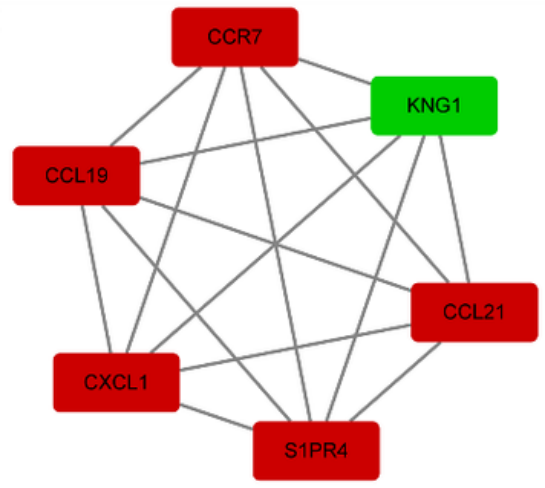

Figure 2

(A) According to the STRING online database, a visual network of 55 nodes and 116 edges was obtained with an interaction score $>0.4$. Nodes represent genes, and edges represent connections between genes. Up-regulated genes are represented in red, whereas downregulated genes are represented in green. (B, C) Two key modules for identifying network gene clustering were identified with MCODE.

A

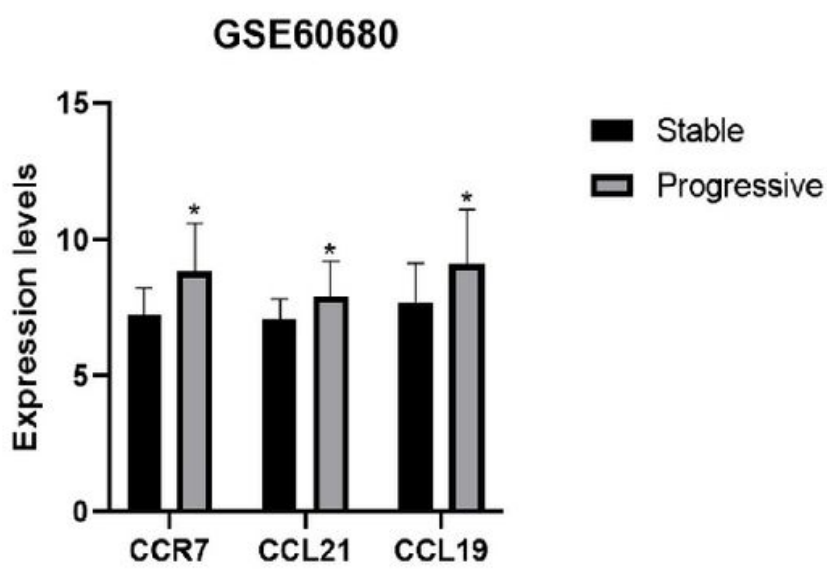

B

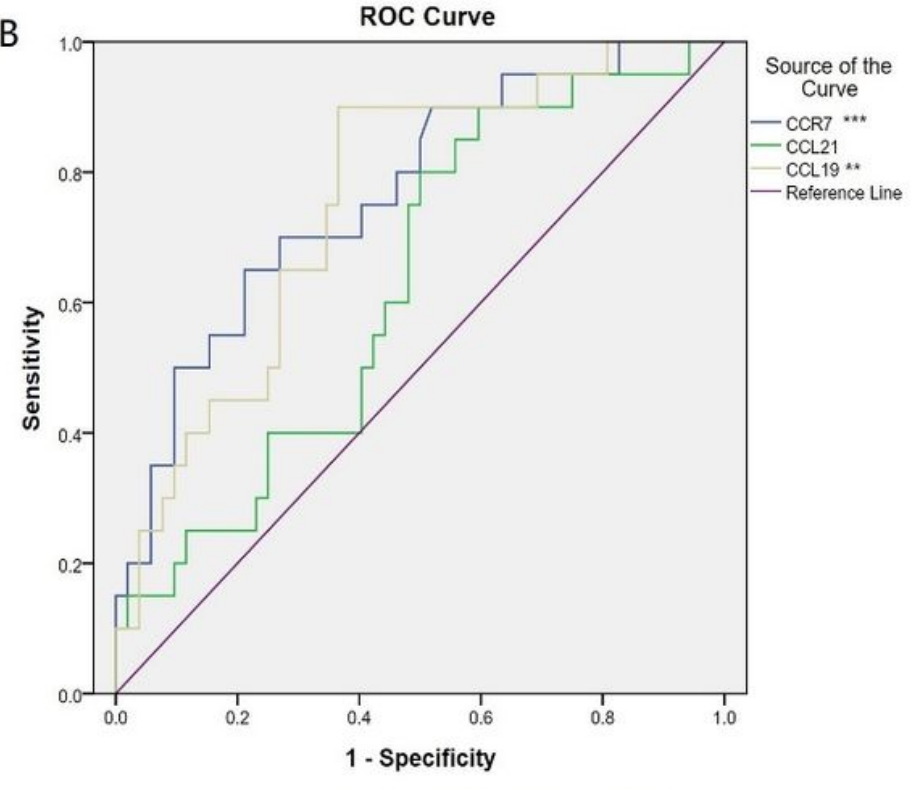

Diagonal segments are produced by ties. 
Figure 3

(A) Expression levels of CCR7, CCL21 and CCL19. (B) ROC curve analysis of CCR7, CCL21 and CCL19 in two data sets. Significance is represented as *, $\mathrm{P}<0.05 ; * \star, \mathrm{P}<0.01 ; * \star *, \mathrm{P}<0.00$.

A
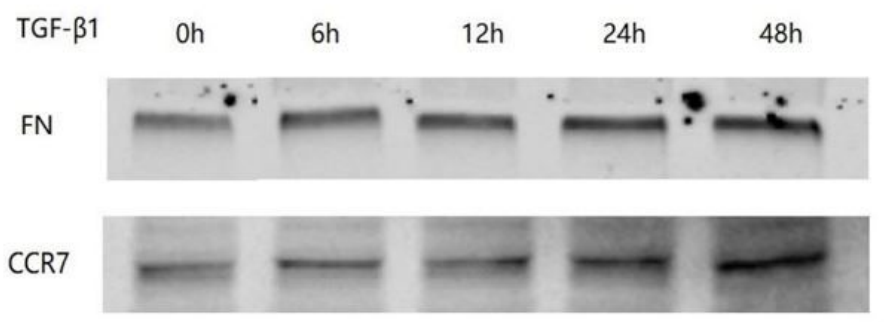

GAPDH

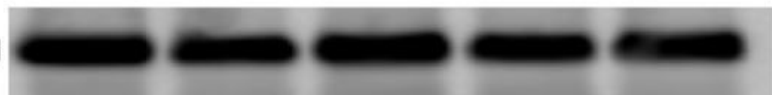

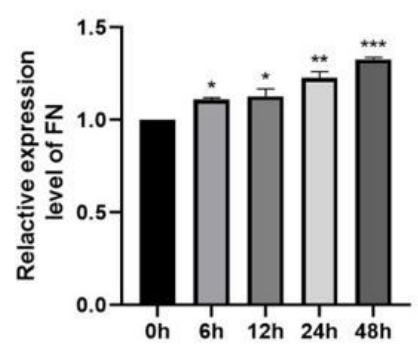

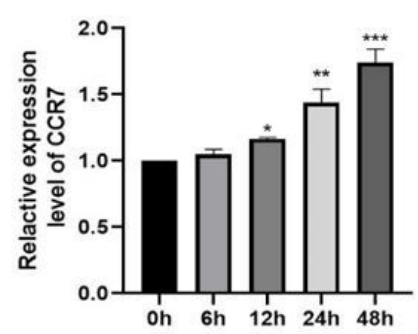

B
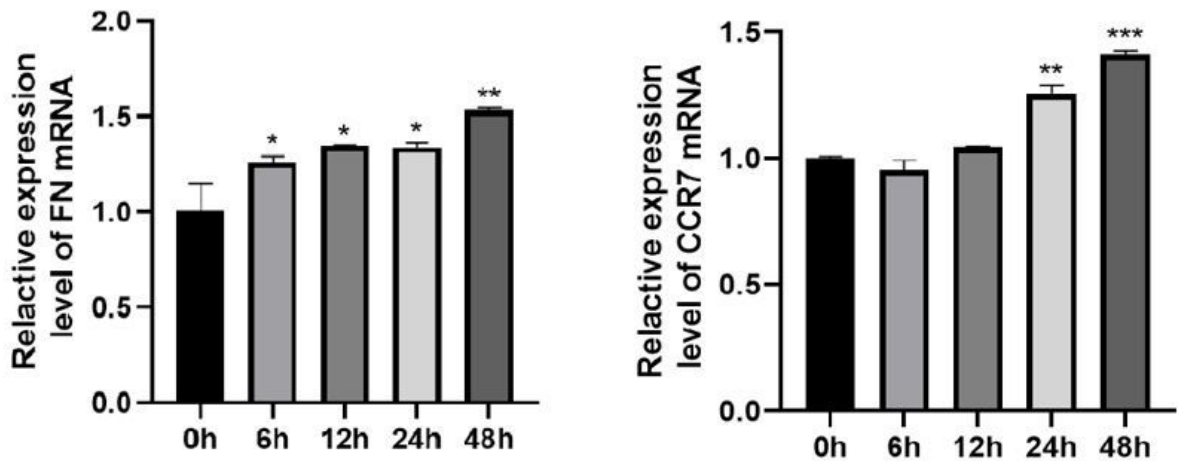

Figure 4

Expression levels of key genes in NRK-52E cells. Cells were treated with $10 \mathrm{ng} / \mathrm{mL}$ TGF- $\beta 1$ for $0,6,12,24$, and 48 hours. (A) FN and CCR7 protein expression levels were analyzed by western blot. (B) FN and CCR7 mRNA levels were analyzed by real-time PCR. Results are presented as the mean \pm standard error of three independent experiments. $\left({ }^{*}, \mathrm{P}<0.05 ;{ }^{* *}, \mathrm{P}<0.01\right.$; ${ }^{* *}, \mathrm{P}<0.001$ vs $\left.0 \mathrm{~h}\right)$ 